\title{
THE TOTAL TORSION ELEMENT GRAPH WITHOUT THE ZERO ELEMENT OF MODULES OVER COMMUTATIVE RINGS
}

\author{
Fatemeh Esmaeili Khalil Saraei
}

\begin{abstract}
Let $M$ be a module over a commutative ring $R$, and let $T(M)$ be its set of torsion elements. The total torsion element graph of $M$ over $R$ is the graph $T(\Gamma(M))$ with vertices all elements of $M$, and two distinct vertices $m$ and $n$ are adjacent if and only if $m+n \in T(M)$. In this paper, we study the basic properties and possible structures of two (induced) subgraphs $\operatorname{Tor}_{0}(\Gamma(M))$ and $T_{0}(\Gamma(M))$ of $T(\Gamma(M))$, with vertices $T(M) \backslash\{0\}$ and $M \backslash\{0\}$, respectively. The main purpose of this paper is to extend the definitions and some results given in [6] to a more general total torsion element graph case.
\end{abstract}

\section{Introduction}

The concept of the graph of the zero-divisors of a ring was first introduced by Beck in [12] when discussing the coloring of a commutative ring. For the vertices of the graph, he takes all elements of a commutative ring $R$ and two distinct vertices $a, b \in R$ are adjacent if $a b=0$. There are many ways to associate a graph to a given ring $R$. The most well-known is certainly the zero-divisor graph $\Gamma(R)$ introduced in [9] whose vertices are the nonzero zerodivisors of $R$. Some properties of this graph may be found in [5] and [10]. In [8], Anderson and Badawi define, for a commutative ring $R$ with nonzero identity, its total graph $\Gamma(R)$. The set of vertices of this graph is $R$ and two different elements $x, y \in R$ are adjacent if and only if $x+y \in Z(R)$ which $Z(R)$ is the set of all zero-divisors of $R$. For a recent generalization of this type of graph see [7] and [11]. Let $M$ be a module over a commutative ring $R$ and let $T(M)$ be the set of all torsion elements of $M$. In [14], the notion of the total torsion element graph of a module over a commutative ring is introduced and denoted by $T(\Gamma(M))$, as the graph with all elements of $M$ as vertices and for distinct $m, n \in M$, the vertices $m$ and $n$ are adjacent if and only if $m+n \in T(M)$. They characterize the girths and diameters of $T(\Gamma(M))$ and two (induced) subgraphs

Received July 27, 2013; Revised October 31, 2013.

2010 Mathematics Subject Classification. Primary 05C99, 13C13.

Key words and phrases. total graph, torsion prime submodule, T-reduced. 
$\operatorname{Tof}(\Gamma(M))$ and $\operatorname{Tor}(\Gamma(M))$ with vertices $\operatorname{Tof}(M)=M \backslash T(M)$ and $T(M)$, respectively. Some other investigation into properties of total torsion element graph of a module over a commutative ring may be found in [1], [2] and [13]. In [6], Anderson and Badawi studied the two subgraphs $Z_{0}(\Gamma(R))$ and $T_{0}(\Gamma(R))$ of $T(\Gamma(R))$, with vertices $Z(R) \backslash\{0\}$ and $R \backslash\{0\}$, respectively. They determined when $Z_{0}(\Gamma(R))$ and $T_{0}(\Gamma(R))$ are connected and computed their diameters and girths.

Throughout this paper all rings are commutative with nonzero identity and all modules are unitary. In this paper, we consider the (induced) subgraphs $\operatorname{Tor}_{0}(\Gamma(M))$ of $\operatorname{Tor}(\Gamma(M))$ and $T_{0}(\Gamma(M))$ of $T(\Gamma(M))$ obtained by deleting 0 as a vertex. Specially, $\operatorname{Tor}_{0}(\Gamma(M))$ (respectively, $T_{0}(\Gamma(M))$ ) has vertices $T(M)^{*}=T(M) \backslash\{0\}$ (respectively, $M^{*}=M \backslash\{0\}$ ), and two distinct vertices $m$ and $n$ are adjacent if and only if $m+n \in T(M)$.

A proper submodule $N$ of an $R$-module $M$ is said to be a prime submodule if whenever $r m \in N$ for some $r \in R, m \in M$, then $m \in N$ or $r M \subseteq N$, so $(N: M)=P$ is a prime ideal of $R$, and $N$ is said to be a $P$-prime submodule (see [15]). The set of all prime submodules of an $R$-module $M$ is denoted by $\operatorname{Spec}_{R}(M)$. In Section 2, we define torsion prime submodules of an $R$-module $M$ by [3, Theorem 3.3]. The intersection of all torsion prime submodules of $M$ denoted by $\operatorname{rad}_{T}(M)$. The study of $\operatorname{Tor}_{0}(\Gamma(M))$ and $T_{0}(\Gamma(M))$ breaks naturally into two cases depending on whether or not $\operatorname{rad}_{T}(M)$ is zero and on the number of torsion prime submodules of $M$. In Sections 3 and 4 , we determine the diameter and girth of subgraph $\operatorname{Tor}_{0}(\Gamma(M))$ of $T_{0}(\Gamma(M))$. In Section 5 , we consider the graph $T_{0}(\Gamma(M))$, show that $\operatorname{diam}\left(T(\Gamma(M))=\operatorname{diam}\left(T_{0}(\Gamma(M))\right)\right.$, when $M$ is a cyclic $R$-module and $|M| \geq 4$ and we determine its girth. Also we show that if $R$ is not an integral domain and $T_{0}(\Gamma(R))$ is connected, then $T_{0}(\Gamma(M))$ is connected for every $R$-module $M$.

For the sake of completeness, we state some definitions and notations used throughout. For a graph $\Gamma$, by $E(\Gamma)$ and $V(\Gamma)$, we denote the set of all edges and vertices, respectively. We recall that a graph is connected if there exists a path connecting any two distinct vertices. The distance between two distinct vertices $x$ and $y$, denoted by $d(x, y)$, is the length of a shortest path connecting them (if such a path does not exist, then $d(x, x)=0$ and $d(x, y)=\infty)$. The diameter of a graph $\Gamma$, denoted by $\operatorname{diam}(\Gamma)$, is equal to $\sup \{d(x, y): x, y \in V(\Gamma)\}$. A graph is complete if it is connected with diameter less than or equal to one. The girth of a graph $\Gamma$, denoted $\operatorname{gr}(\Gamma)$, is the length of a shortest cycle in $\Gamma$, provided $\Gamma$ contains a cycle; otherwise; $\operatorname{gr}(\Gamma)=\infty$. We denote the complete graph on $n$ vertices by $K^{n}$ and the complete bipartite graph on $m$ and $n$ vertices by $K^{m, n}$ (we allow $m$ and $n$ to be infinite cardinals).

\section{Torsion subset of a module}

We devote this section to the set of torsion elements of an $R$-module $M$. We define torsion prime submodules of $M$ and collect some basic properties of 
them which we will use throughout this paper. We first begin with the following result that is proved in ([3, Theorem 3.3]).

Theorem 2.1. Let $M$ be a module over a commutative ring $R$ and $T(M) \neq M$. Then $T(M)$ is a union of prime submodules of $M$ (see [3, Theorem 3.3]).

Corollary 2.2. Let $M$ be a module over a commutative ring $R$ and $T(M) \neq M$. Then $\operatorname{Spec}_{R}(M) \neq \emptyset$.

Proof. It is clear by Theorem 2.1.

Definition. Let $M$ be an $R$-module. A prime submodule $L$ of $M$ is called a torsion prime submodule when, $L \subseteq T(M)$. Assume that $\operatorname{Spec}_{T}(M)$ denotes all torsion prime submodules of $M$. It is clear that $\operatorname{Spec}_{T}(M) \subseteq \operatorname{Spec}(M)$ and if $T(M) \neq M$, then $\operatorname{Spec}_{T}(M) \neq \emptyset$ and $T(M)=\bigcup_{L \in \operatorname{Spec}_{T}(M)} L$ by Theorem 2.1. The $T$-radical of $M$, denoted by $\operatorname{rad}_{T}(M)$, is defined to be the intersection of all torsion prime submodules of $M$. If there is no torsion prime submodule of $M$, then we put $\operatorname{rad}_{T}(M)=M$. An $R$-module $M$ is called $T$-reduced, if $\operatorname{rad}_{T}(M)=0$.

We have the following lemma containing several results which we will use in throughout this paper.

Lemma 2.3. Let $M$ be an $R$-module and $T(M) \neq M$. Then:

(1) $(T(M): M)=Z(R)$.

(2) If $L \in \operatorname{Spec}_{T}(M)$ is a P-prime submodule, then $P \subseteq Z(R)$.

(3) If $x \in T(M)$ and $y \in \operatorname{rad}(M)$, then $x+y \in T(M)$.

(4) If $0 \in \operatorname{Spec}_{T}(M)$, then $T(M)=0$.

(5) $r \in Z(R)$ if and only if $r m \in T(M)$ for all $m \in M$.

Proof. (1) It follows from [2, Lemma 2.5].

(2) Assume that $L \in \operatorname{Spec}_{T}(M)$ is a $P$-prime submodule. So $(L: M)=P$ and $P M \subseteq L \subseteq T(M)$. Then $P \subseteq(T(M): M)=Z(R)$.

(3) Let $x \in T(M)$. Then $x \in L \subseteq T(M)$ for some $L \in \operatorname{Spec}_{T}(M)$ by Theorem 2.1. Since $y \in \operatorname{rad}(M) \subseteq L$, it follows that $x+y \in L \subseteq T(M)$.

(4) Since $T(M) \neq M$, so $\left(0:_{R} M\right)=0$. If $m \in T(M)$, then $r m=0$ for some nonzero element $r \in R$. So $m=0$, since 0 is a prime submodule of $M$.

(5) Assume that $r \in Z(R)$ and $m \in M$. Then $r s=0$ for some nonzero element $s \in R$, so $s(r m)=0$. Thus $r m \in T(M)$. Conversely, suppose that $x \in M \backslash T(M)$, then $r x \in T(M)$. So $t(r x)=0$ for some nonzero element $t \in R$. If $t r \neq 0$, then $x \in T(M)$ which is a contradiction. Thus $t r=0$ as required.

Proposition 2.4. Let $M$ be a module over a commutative ring $R$ with $T(M) \neq$ $M$ such that $R$ is not an integral domain. If $L_{1} \cap L_{2}=0$ for some $L_{1}, L_{2} \in$ $\operatorname{Spec}_{T}(M)$, then:

(1) Either $K \cap L_{1} \neq 0$ or $K \cap L_{2} \neq 0$ for every $K \in \operatorname{Spec}(M)$.

(2) If $K \cap L_{1} \neq 0$ and $K \cap L_{2}=0$ for some $K \in \operatorname{Spec}(M)$, then $\left(L_{1}: M\right)=$ $(K: M)$. 
Proof. (1) Let $L_{1}$ be a $P_{1}$ - prime submodule and $L_{2}$ be a $P_{2^{-}}$prime submodule of $M$. Since $Z(R) \neq 0$, so $P_{1}, P_{2} \neq 0$ by Lemma 2.3. Therefore $P_{1} P_{2} M \subseteq$ $P_{1} M \cap P_{2} M \subseteq L_{1} \cap L_{2}=0$. Now, suppose that $K$ is a $P$-prime submodule of $M$. Thus $P_{1} P_{2} M=0 \subseteq K$. This implies that either $P_{1} M \subseteq K$ or $P_{2} M \subseteq K$, since $K$ is a prime submodule of $M$. Hence either $0 \neq P_{1} M \subseteq K \cap L_{1}$ or $0 \neq P_{2} M \subseteq K \cap L_{2}$.

(2) Let $L_{1}$ be a $P_{1}$ - prime submodule and $K$ be a $P$-prime submodule of $M$. We must show that $P_{1}=P$. By (1), it is clear that $P_{1} \subseteq P$. On the other hand $P P_{2} M \subseteq P M \cap P_{2} M \subseteq K \cap L_{2}=0$. Then $P P_{2} M=0 \subseteq L_{1}$. So $P \subseteq P_{1}$, since $L_{1}$ is a $P_{1}$ - prime submodule and $L_{1} \cap L_{2}=0$.

\section{The diameter of $\operatorname{Tor}_{0}(\Gamma(M))$}

In this section, we compute the diameter of graph $\operatorname{Tor}_{0}(\Gamma(M))$. Our answers depend on whether or not $M$ is a $T$-reduced module. It is clear that $T(M)=M$ if and only if graph $T_{0}(\Gamma(M))$ is complete and $T_{0}(\Gamma(M))$ is disconnected when $T(M)=0$ and $M \neq 0$. So we may assume that $T(M) \neq 0$ and $T(M) \neq M$.

We first study the case when $M$ is not a $T$-reduced $R$-module.

Theorem 3.1. Let $M$ be a non-T-reduced $R$-module. Then Tor $_{0}(\Gamma(M))$ is connected with $\operatorname{diam}\left(\operatorname{Tor}_{0}(\Gamma(M))\right) \in\{0,1,2\}$.

Proof. Assume that $M$ is a non-T-reduced $R$-module. Let $m, m^{\prime} \in T(M)^{*}$ be distinct vertices of $\operatorname{Tor}_{0}(\Gamma(M))$. If either $m \in \operatorname{rad}_{T}(M)$ or $m^{\prime} \in \operatorname{rad}_{T}(M)$, then $m+m^{\prime} \in T(M)$ by Lemma 2.3. So, $m-m^{\prime}$ is an edge in $\operatorname{Tor}_{0}(\Gamma(M))$. Therefore, we may assume that $m \notin \operatorname{rad}_{T}(M), m^{\prime} \notin \operatorname{rad}_{T}(M)$ and $m+m^{\prime} \notin T(M)$. Let $0 \neq n \in \operatorname{rad}_{T}(M)$. Then $m-n-m^{\prime}$ is a path in $\operatorname{Tor}_{0}(\Gamma(M))$ of length 2 by Lemma 2.3 and hence $\operatorname{diam}\left(\operatorname{Tor}_{0}(\Gamma(M))\right) \leq 2$.

Note that $\operatorname{Tor}_{0}(\Gamma(M))$ is a complete graph if and only if $T(M)$ is a submodule of $M$, and so $\operatorname{diam}\left(\operatorname{Tor}_{0}(\Gamma(M))\right) \leq 1$. Also, $T(M)$ is a union of torsion prime submodules of $M$ by Theorem 2.1. So, if $M$ is a non- $T$-reduced $R$-module with $T(M) \neq M$, then $\operatorname{diam}\left(\operatorname{Tor}_{0}(\Gamma(M))\right)=0$ if and only if $\left|T(M)^{*}\right|=1$.

We next consider when $M$ is a $T$-reduced $R$-module. It is clear that, $M$ is a torsion free $R$-module if and only if $\left|\operatorname{Spec}_{T}(M)\right|=1$. If $M$ is a torsion free $R$ module, then $\operatorname{Tor}_{0}(\Gamma(M))$ is the empty graph; so we assume that $\left|\operatorname{Spec}_{T}(M)\right| \geq$ 2 .

Proposition 3.2. Let $M$ be a $T$-reduced $R$-module with $\left|\operatorname{Spec}_{T}(M)\right|=2$ and $T(M) \notin \operatorname{Spec}_{T}(M)$. Then $\operatorname{Tor}_{0}(\Gamma(M))$ is not connected.

Proof. Suppose that $\left|\operatorname{Spec}_{T}(M)\right|=2$. Let $L_{1}, L_{2} \in \operatorname{Spec}_{T}(M)$. So, $L_{1} \cap L_{2}=$ $\operatorname{rad}_{T}(M)=0$ and $T(M)=L_{1} \cup L_{2}$ by Theorem 2.1. Let $0 \neq m \in L_{1}$ and $0 \neq n \in L_{2}$. Then $m+n \notin T(M)$, since $L_{1} \cap L_{2}=0$. So there can be no path in $\operatorname{Tor}_{0}(\Gamma(M))$ from any $L_{1}^{*}$ to any $L_{2}^{*}$. Thus $\operatorname{Tor}_{0}(\Gamma(M))$ is not connected.

Definition. Let $M$ be an $R$-module and $m, m^{\prime} \in M$ be nonzero distinct elements. We say that $m-x_{1}-\cdots-x_{n}-m^{\prime}$ is a torsion path from $m$ to 
$m^{\prime}$ if $x_{1}, \ldots, x_{n} \in T(M)^{*}$ and $x_{i}+x_{i+1} \in T(M)$ for every $0 \leq i \leq n$ (let $x_{0}=m$ and $\left.x_{n+1}=m^{\prime}\right)$. We define the torsion distance between two distinct vertices $m$ and $m^{\prime}$, denoted by $\mathrm{d}_{\mathrm{T}}\left(m, m^{\prime}\right)$, to be the length of a shortest torsion path connecting them (if such a path does not exist, then $d_{T}(m, m)=0$ and $\left.d_{T}\left(m, m^{\prime}\right)=\infty\right)$.

Definition. Let $L$ and $K$ be two distinct torsion prime submodules of an $R$ module $M$. We say that there is a torsion path between $L$ and $K$, if there exists a torsion path from $x$ to $y$ for some $x \in L^{*}$ and $y \in K^{*}$. We define the torsion distance between two distinct torsion prime submodules $L$ and $K$, denoted by $\mathrm{d}_{\mathrm{T}}(L, K)$, to be the length of a shortest torsion path connecting them (if such a path does not exist, then $\left.d_{T}(L, K)=\infty\right)$. It is clear that $d_{T}(L, K)=0$, when $L \cap K \neq 0$.

We obtain some results concerning $\operatorname{Tor}_{0}(\Gamma(M))$ and it's torsion paths by the following lemma.

Lemma 3.3. Let $M$ be an $R$-module with $T(M) \neq M$ such that $R$ is not an integral domain. If there is a torsion path between $L_{1}$ and $L_{2}$ for some torsion prime submodules $L_{1}$ and $L_{2}$ of $M$, then $\mathrm{d}_{\mathrm{T}}\left(L_{1}, L_{2}\right) \leq 3$.

Proof. If $L_{1} \cap L_{2} \neq 0$, then it is clear that $\mathrm{d}_{\mathrm{T}}\left(L_{1}, L_{2}\right)=0$. So we may assume that $L_{1} \cap L_{2}=0$. Suppose that there is a shortest path $x-t_{1}-t_{2}-\cdots-t_{n-1}-y$ from $x$ to $y$ for some $x \in L_{1}^{*}$ and $y \in L_{2}^{*}$ of length $n$. So $t_{i} \notin L_{1}$ and $t_{i} \notin L_{2}$ for each $i=1, \ldots, n-1$. Since $t_{1} \in T(M)$, so $t_{1} \in L_{3}$ for some torsion prime submodule $L_{3}$ of $M$ by Theorem 2.1. Therefore either $L_{3} \cap L_{2} \neq 0$ or $L_{3} \cap L_{1} \neq 0$ by Proposition 2.4. If $0 \neq a \in L_{3} \cap L_{2}$, then $x-t_{1}-a$ is a torsion path between $L_{1}$ and $L_{2}$ and so $\mathrm{d}_{\mathrm{T}}\left(L_{1}, L_{2}\right) \leq 2$, since $L_{1} \cap L_{2}=0$. Now, suppose that $0 \neq b \in L_{3} \cap L_{1}$. Then $b-t_{1}-t_{2}-\cdots-t_{n-1}-y$ is a torsion path between $L_{1}$ and $L_{2}$. If $t_{2} \in L_{3}$, then $b-t_{2}-\cdots-t_{n-1}-y$ is a torsion path between $L_{1}$ and $L_{2}$ of length $n-1$. If $t_{2} \notin L_{3}$, then $t_{2} \in L_{4}$ for some torsion prime submodule $L_{4}$ of $M$. Similarly, either $L_{4} \cap L_{2} \neq 0$ or $L_{4} \cap L_{1} \neq 0$ by Proposition 2.4. If $0 \neq c \in L_{4} \cap L_{2}$, then $x-t_{1}-t_{2}-c$ is a torsion path between $L_{1}$ and $L_{2}$ and so $\mathrm{d}_{\mathrm{T}}\left(L_{1}, L_{2}\right) \leq 3$. Otherwise there is a nonzero element $d \in L_{4} \cap L_{1}$. Thus $d-t_{2}-t_{3}-\cdots-t_{n-1}-y$ is a torsion path between $L_{1}$ and $L_{2}$ of length $n-1$. By similar argument to the above on $t_{i}$ 's for $i=2, \ldots, n-1$, we conclude that $\mathrm{d}_{\mathrm{T}}\left(L_{1}, L_{2}\right) \leq 3$.

Theorem 3.4. Let $M$ be a T-reduced $R$-module and $T(M) \neq M$. Then $\operatorname{diam}\left(\operatorname{Tor}_{0}(\Gamma(M))\right) \in\{1,2,3,4,5, \infty\}$.

Proof. If $R$ is an integral domain, then it is clear that $T(M)$ is a prime submodule of $M$ and $\operatorname{diam}\left(\operatorname{Tor}_{0}(M)\right) \in\{0,1\}$. So we assume that $R$ is not an integral domain. Let $M$ be a $T$-reduced $R$-module and $x, y \in T(M)^{*}$. If $x, y \in L$ for some torsion prime submodule $L$ of $M$, then it is clear that $d(x, y)=1$. So we may assume that $x \in L_{1}$ and $y \in L_{2}$ for some torsion prime submodules $L_{1}$ and $L_{2}$ of $M$. If $\mathrm{d}_{\mathrm{T}}\left(L_{1}, L_{2}\right)=\infty$, then $d(x, y)=\infty=\operatorname{diam}\left(\operatorname{Tor}_{0}(\Gamma(M))\right)$. So 
suppose that $\mathrm{d}_{\mathrm{T}}\left(L_{1}, L_{2}\right)<\infty$. Thus $\mathrm{d}_{\mathrm{T}}\left(L_{1}, L_{2}\right) \leq 3$ by Lemma 3.3. Now we consider the following four cases:

Case 1. If $\mathrm{d}_{\mathrm{T}}\left(L_{1}, L_{2}\right)=0$, then there is a nonzero element $a \in L_{1} \cap L_{2}$. So $x-a-y$ is a torsion path and so $d(x, y)=2$.

Case 2. If $\mathrm{d}_{\mathrm{T}}\left(L_{1}, L_{2}\right)=1$, then $m+n \in T(M)$ for some $m \in L_{1}$ and $n \in L_{2}$. Thus $x-m-n-y$ is a path in $\operatorname{Tor}_{0}(\Gamma(M))$. If either $x=m$ or $y=n$, then $d(x, y)=1$ or 2 , otherwise $d(x, y)=3$.

Case 3. If $\mathrm{d}_{\mathrm{T}}\left(L_{1}, L_{2}\right)=2$, then $m-k-n$ is a torsion path from $m$ to $n$ for some $m \in L_{1}, n \in L_{2}$ and $k \in T(M)^{*}$. Therefore we have the torsion path $x-m-k-n-y$. Hence by the various possibility for the $x$ and $y, d(x, y)=2,3$ or 4 .

Case 4. If $\mathrm{d}_{\mathrm{T}}\left(L_{1}, L_{2}\right)=3$, then $m-k-g-n$ is a torsion path from $m$ to $n$ for some $m \in L_{1}, n \in L_{2}$ and $k, g \in T(M)^{*}$. Therefore we have the torsion path $x-m-k-g-n-y$. Hence by the various possibility for the $x$ and $y$, $d(x, y)=3,4$ or 5 . Then $\operatorname{diam}\left(\operatorname{Tor}_{0}(\Gamma(M))\right) \in\{1,2,3,4,5, \infty\}$.

\section{The girth of $\operatorname{Tor}_{0}(\Gamma(M))$}

The aim of this section is to show that $\operatorname{gr}\left(\operatorname{Tor}_{0}(\Gamma(M))\right) \in\{3, \infty\}$. Our answer depends on the number of torsion prime submodules of $M$.

We first consider the case when $M$ is not a $T$-reduced $R$-module.

Theorem 4.1. Let $M$ be a non-T-reduced $R$-module and $T(M) \neq M$. Then $\operatorname{gr}\left(\operatorname{Tor}_{0}(\Gamma(M))\right)=\infty$ if and only if $M$ has a unique nonzero torsion prime submodule $L$ with $T(M)=L$ and $|L| \leq 3$. Otherwise gr $\left(\operatorname{Tor}_{0}(\Gamma(M))\right)=3$.

Proof. Suppose that $\left|\operatorname{Spec}_{T}(M)\right| \geq 2$. Let $L_{1}$ and $L_{2}$ be distinct torsion prime submodules of $M$. So $L_{1}, L_{2} \subseteq T(M)$. Then $0 \neq \operatorname{rad}_{T}(M) \subseteq L_{1} \cap L_{2} \subset L_{1}$, since $\operatorname{rad}_{T}(M) \neq 0$. So $\left|L_{1} \cap L_{2}\right| \geq 2$ and thus $\left|L_{1}\right| \geq 4$. Let $x, y, z \in L_{1}^{*}$ be distinct. Then $x-y-z-x$ is a 3 -cycle in $\operatorname{Tor}_{0}(\Gamma(M))$. So $\operatorname{gr}\left(\operatorname{Tor}_{0}(\Gamma(M))\right)=3$. Now, suppose that $\operatorname{Spec}_{T}(M)=\{L\}$ and thus $T(M)=L$ by Theorem 2.1. So $\operatorname{gr}\left(\operatorname{Tor}_{0}(\Gamma(M))\right)=3$ if and only if $|T(M)| \geq 4$. If $|T(M)| \leq 3$, it is clear that $\operatorname{gr}\left(\operatorname{Tor}_{0}(\Gamma(M))\right)=\infty$.

We next handle the case when $M$ is a $T$-reduced $R$-module.

Theorem 4.2. Let $M$ be a $T$-reduced $R$-module and $T(M) \neq 0, M$. Then $\operatorname{gr}\left(\operatorname{Tor}_{0}(\Gamma(M))\right)=\infty$ if and only if $\operatorname{Spec}_{T}(M)=\left\{L_{1}, L_{2}\right\}$ with $\max \left\{\left|L_{1}\right|,\left|L_{2}\right|\right\}$ $\leq 3$. Otherwise, $\operatorname{gr}\left(\operatorname{Tor}_{0}(\Gamma(M))\right)=3$.

Proof. Suppose that $L_{1}, L_{2}$ and $L_{3}$ are distinct torsion prime submodule of $M$. By Proposition 2.4, we may assume that $L_{1} \cap L_{2} \neq 0$, then $\left|L_{1} \cap L_{2}\right| \geq 2$ and $\left|L_{1}\right| \geq 4$. Let $m_{1}, m_{2}, m_{3} \in L_{1}^{*}$ be distinct. Then $m_{1}-m_{2}-m_{3}-m_{1}$ is a 3 -cycle in $\operatorname{Tor}_{0}(\Gamma(M))$. Thus $\operatorname{gr}\left(\operatorname{Tor}_{0}(\Gamma(M))\right)=3$, if $\left|\operatorname{Spec}_{T}(M)\right| \geq 3$.

So, we may assume that $\operatorname{Spec}_{T}(M)=\{K, L\}$. Therefore $T(M)=K \cup L$ and $K \cap L=\{0\}$. So no $x \in K^{*}$ and $y \in L^{*}$ are adjacent in $\operatorname{Tor}_{0}(\Gamma(M))$ by Proposition 3.2. Thus $\operatorname{gr}\left(\operatorname{Tor}_{0}(\Gamma(M))\right)=3$ if and only if $|K| \geq 4$ or $|L| \geq 4$. Otherwise $\operatorname{gr}\left(\operatorname{Tor}_{0}(\Gamma(M))\right)=\infty$. 
We end this section with the following example.

Example 4.3. (1) Let $R=Z_{6}$ and $M=R$ as an $R$-module. Then $T(M)=$ $\{\overline{0}, \overline{2}, \overline{3}, \overline{4}\}$ and $\operatorname{Spec}(M)=\operatorname{Spec}_{T}(M)=\{\langle\overline{2}\rangle,\langle\overline{3}\rangle\}$. It is clear that $M$ is a $T$-reduced $R$-module and $T(M)=\langle\overline{2}\rangle \cup\langle\overline{3}\rangle$. So, Tor $_{0}(\Gamma(M))$ is not connected with $\operatorname{diam}\left(\operatorname{Tor}_{0}(\Gamma(M))\right)=\infty$ and $\operatorname{gr}\left(\operatorname{Tor}_{0}(\Gamma(M))\right)=\infty$.

(2) Let $R=Z_{60}$ and $M=R$ as an $R$-module. Then $\operatorname{Spec}(M)=\operatorname{Spec}_{T}(M)=$ $\{\langle\overline{2}\rangle,\langle\overline{3}\rangle,\langle\overline{5}\rangle\}$ and $\operatorname{rad}_{T}(M)=\langle\overline{30}\rangle$. So $M$ is a non- $T$-reduced $R$-module and $T(M)=\langle\overline{2}\rangle \cup\langle\overline{3}\rangle \cup\langle\overline{5}\rangle$. It is clear that $\operatorname{Tor}_{0}(\Gamma(M))$ is connected with $\operatorname{diam}\left(\operatorname{Tor}_{0}(\Gamma(M))\right)=2$ and $\operatorname{gr}\left(\operatorname{Tor}_{0}(\Gamma(M))\right)=3$.

\section{5. $T_{0}(\Gamma(M))$}

In this section, we state a general structure for $T_{0}(\Gamma(M))$. We explicitly compute $\operatorname{gr}\left(T_{0}(\Gamma(M))\right)$. Also, we investigate the relationship of diameters and girths between $T(\Gamma(M))$ and $T_{0}(\Gamma(M))$ with assumption that $M$ is a cyclic $R$-module.

Assume that $d_{T_{0}}(m, n)$ denotes the distance from $m$ to $n$ in $T_{0}(\Gamma(M))$ for $0 \neq m, n \in M$. We first show that $d_{T_{0}}(m, n)=d_{T}(m, n)$. We begin with the following lemma.

Lemma 5.1. Let $M$ an $R$-module and $0 \neq m, n \in M$. Then $m, n$ are connected by a path in $T_{0}(\Gamma(M))$ if and only if $m, n$ are connected by a path in $T(\Gamma(M))$. Moreover, $d_{T_{0}}(m, n)=d_{T}(m, n)$ and $\operatorname{diam}\left(T_{0}(\Gamma(M))\right) \leq \operatorname{diam}(T(\Gamma(M)))$.

Proof. If $m, n$ are connected by a path in $T_{0}(\Gamma(M))$, then it is clear that they are connected by a path in $T(\Gamma(M))$. Conversely, suppose that $m-x_{1}-x_{2}-\cdots-$ $x_{k}-n$ is a shortest path from $m$ to $n$ in $T(\Gamma(M))$. If $x_{i}=0$ for some $1 \leq i \leq k$, then $x_{i-1}, x_{i+1} \in T(M)^{*}$ and $x_{i-1}+x_{i+1} \in \operatorname{Tof}(M)$ (set $x_{0}=m$ and $x_{k+1}=$ $n)$. Let $x_{i}^{\prime}=-\left(x_{i-1}+x_{i+1}\right)$. Then $m-x_{1}-\cdots-x_{i-1}-x_{i}^{\prime}-x_{i+1}-\cdots-x_{k}-n$ is a shortest path from $m$ to $n$ in $T_{0}(\Gamma(M))$. The "moreover" statement is clear.

Recall that if $T(M)$ is a submodule of $M$, then $T(\Gamma(M))$ is not connected [14, Theorem 2.1]. If $T(M)$ is not a submodule of $M$, then $T(\Gamma(M))$ is connected if and only if $M=\langle T(M)\rangle[14$, Theorem 3.2] and $\operatorname{diam}(T(\Gamma(M))) \leq n$, where $n \geq 2$ is the least positive integer such that $M=\left\langle m_{1}, m_{2}, \ldots, m_{n}\right\rangle$ for some $m_{1}, \ldots, m_{n} \in T(M)[14$, Theorem 3.3].

Also, if $M$ is a cyclic $R$-module with generator $m$, then $\operatorname{diam}(T(\Gamma(M)))=$ $d(0, m)[14$, Theorem 3.4]. The following example show that for an $R$-module $M$, the structures of $T(\Gamma(M))$ and $T_{0}(\Gamma(M))$ are not independent of ring $R$.

Example 5.2. (1) Let $R=Z$ be the integer numbers ring and $M=Z_{2}$ as an $R$-module. It is clear that $T(M)=M$ and easily verified that $T_{0}(\Gamma(M))$ and $T(\Gamma(M))$ are connected.

(2) Let $R=Z_{2}$ and $M=R$ as an $R$-module. Then $T_{0}(\Gamma(M))$ is connected but $T(\Gamma(M))$ is not connected by [6, Theorem 4.2$]$. 
Now, we obtain various results for $\operatorname{diam}(T(\Gamma(M)))$ and $\operatorname{diam}\left(T_{0}(\Gamma(M))\right)$ when $|M| \leq 3$ by Theorem 5.3 and Theorem 5.4 .

Theorem 5.3. Let $M$ be a module over a commutative ring $R$ and $|M|=2$.

(1) If $M=T(M)$, then $\operatorname{diam}\left(T_{0}(\Gamma(M))\right)=0$ and $\operatorname{diam}(T(\Gamma(M)))=1$.

(2) If $M \neq T(M)$, then $\operatorname{diam}\left(T_{0}(\Gamma(M))\right)=0$ and $\operatorname{diam}(T(\Gamma(M)))=\infty$.

Proof. The proof is clear.

Theorem 5.4. Let $M$ be a module over a commutative ring $R$ and $|M|=3$.

(1) If $M=T(M)$, then $\operatorname{diam}\left(T_{0}(\Gamma(M))\right)=\operatorname{diam}(T(\Gamma(M)))=1$.

(2) If $M \neq T(M)$ and $|T(M)|=1$, then $\operatorname{diam}\left(T_{0}(\Gamma(M))\right)=1$ and $\operatorname{diam}(T(\Gamma(M)))=\infty$.

(3) If $M \neq T(M)$ and $|T(M)|=2$, then $\operatorname{diam}\left(T_{0}(\Gamma(M))\right)=1$ and $\operatorname{diam}(T(\Gamma(M)))=2$.

Proof. Let $|M|=3$. Then $M=\{0, x, y\}$ with $x+y=0,2 x=y$ and $2 y=x$.

(1) If $M=T(M)$, then it is clear that $\operatorname{diam}\left(T_{0}(\Gamma(M))\right)=\operatorname{diam}(T(\Gamma(M)))=$ 1.

(2) If $M \neq T(M)$ and $|T(M)|=1$, then $T(M)=\{0\}$. So $\operatorname{diam}\left(T_{0}(\Gamma(M))\right)=$ 1 but $x$ and $y$ are not adjacent to 0 in $T(\Gamma(M))$. Thus $\operatorname{diam}(T(\Gamma(M)))=\infty$.

(3) If $M \neq T(M)$ and $|T(M)|=2$, then we may assume that $T(M)=\{0, x\}$. So $\operatorname{diam}\left(T_{0}(\Gamma(M))\right)=1$ and $0-x-y$ is a path from 0 to $y$ in $T(\Gamma(M))$. Thus $\operatorname{diam}(T(\Gamma(M)))=2$.

Corollary 5.5. Let $M$ be a module over a commutative ring $R$ and $|M| \leq 3$.

(1) If $M=T(M)$, then $T_{0}(\Gamma(M))$ and $T(\Gamma(M))$ are connected.

(2) If $|T(M)|=1$, then $T_{0}(\Gamma(M))$ is connected, but $T(\Gamma(M))$ is not connected.

(3) If $|M|=3$ and $|T(M)|=2$, then $T_{0}(\Gamma(M))$ and $T(\Gamma(M))$ are connected. Proof. The proof is clear by Theorem 5.3 and Theorem 5.4.

Theorem 5.6. Let $M$ be a module over a commutative ring $R$. If $|M| \geq 4$, then $T_{0}(\Gamma(M))$ is connected if and only if $T(\Gamma(M))$ is connected.

Proof. Suppose that $T(\Gamma(M))$ is connected. Then $T_{0}(\Gamma(M))$ is also connected by Lemma 5.1. Conversely, suppose that $T_{0}(\Gamma(M))$ is connected and $|M| \geq 4$. So $M$ is not a torsion-free module and there is an $m \in T(M)^{*}$. Let $0 \neq n \in M$. Then there is a path from $m$ to $n$ in $T_{0}(\Gamma(M))$. Since $m \in T(M)$, so $m$ is adjacent to 0 in $T(\Gamma(M))$. Hence there is a path from $n$ to 0 in $T(\Gamma(M))$ and thus $T(\Gamma(M))$ is also connected.

Example 5.7. Let $R=Z_{6}$ and $M=R$ as an $R$-module. By Example 4.3, we have $\operatorname{diam}\left(\operatorname{Tor}_{0}(\Gamma(M))\right)=\infty$ and $\operatorname{diam}\left(T_{0}(\Gamma(M))\right)=2$. Also, If $R=M=$ $Z_{60}$, then $\operatorname{diam}\left(T_{0}(\Gamma(M))\right)=\operatorname{diam}\left(\operatorname{Tor}_{0}(\Gamma(M))\right)=2$ and $\operatorname{diam}\left(\operatorname{Tor}_{0}(\Gamma(M))\right)$ $=0<\operatorname{diam}\left(T_{0}(\Gamma(M))\right)=\infty$ for $R=M=Z_{4}$. Therefore there is no relationship between $\operatorname{diam}\left(\operatorname{Tor}_{0}(\Gamma(M))\right)$ and $\operatorname{diam}\left(T_{0}(\Gamma(M))\right)$. 
Our next goal is to show that $\operatorname{diam}\left(T_{0}(\Gamma(M))\right)=\operatorname{diam}(T(\Gamma(M)))$ when $M$ is a cyclic $R$-module with $|M| \geq 4$.

We begin with the following theorem.

Theorem 5.8. Let $M$ be a cyclic $R$-module and $T(M) \neq 0$.

(1) $T(\Gamma(M))$ is connected if and only if $\operatorname{diam}(T(\Gamma(M)))<\infty$.

(2) $T_{0}(\Gamma(M))$ is connected if and only if $\operatorname{diam}\left(T_{0}(\Gamma(M))\right)<\infty$.

Proof. Let $M$ be a cyclic $R$-module with generator $m$ and $T(M) \neq 0$.

(1) Suppose that $T(\Gamma(M))$ is connected. So there is a finite path $0-x_{1}-$ $\cdots-x_{k-1}-m$ from 0 to $m$. Then $\operatorname{diam}(T(\Gamma(M)))=d(0, m)=k<\infty$ by $[14$, Theorem 3.4]. The converse is clear.

(2) Suppose that $T_{0}(\Gamma(M))$ is connected. So there is an element $a \in T(M)^{*}$. Let $0 \neq b \in M$. Then there is a path from $a$ to $b$ in $T_{0}(\Gamma(M))$. But $a$ is adjacent to 0 in $T(\Gamma(M))$; therefore there is a path from 0 to $b$ in $T(\Gamma(M))$ and $T(\Gamma(M))$ is connected. So $\operatorname{diam}\left(T_{0}(\Gamma(M))\right) \leq \operatorname{diam}(T(\Gamma(M)))<\infty$ by part (1) and Lemma 5.1.

The converse is clear.

Lemma 5.9. Let $M$ be a cyclic module over a commutative ring $R$ with generator $m$. Let $n \geq 2$ be the least integer such that $M=\left\langle m_{1}, m_{2}, \ldots, m_{n}\right\rangle$ for some $m_{1}, \ldots, m_{n} \in T(M)$. If $0 \neq x \in M$, then:

(1) If $x \in T(M)^{*}$, then $d_{T_{0}}(m, x)=d_{T}(m, x) \in\{n-1, n\}$.

(2) If $n$ is an even integer, then $d_{T_{0}}(m-x, x)=k=d_{T}(m-x, x)$ for some even integer $k \leq n$.

(3) If $n$ is an odd integer and $m \neq-x$, then $d_{T_{0}}(m+x, x)=k=d_{T}(m+x, x)$ for some odd integer $k \leq n$.

(4) If $n$ is an even integer, then $d_{T_{0}}(m-x, x)=n=d_{T}(m-x, x)$ for every $x \in T(M)^{*}$.

(5) If $n$ is an odd integer, then $d_{T_{0}}(m+x, x)=n=d_{T}(m+x, x)$ for every $x \in T(M)^{*}$.

Proof. It is clear that $2 \leq \operatorname{diam}(T(\Gamma(M)))=n<\infty$ by [14, Theorem 3.3].

(1) Let $x-y_{1}-y_{2}-\cdots-y_{k-1}-m$ be a shortest path from $x$ to $m$ in $T_{0}(\Gamma(M))$ of length $k$. Then $k=d_{T_{0}}(x, m)=d_{T}(x, m) \leq n$ by Lemma 5.1. Thus $x+y_{1}, y_{1}+y_{2}, \ldots, y_{k+1}+m \in T(M)$. Since $m \in\left\langle x, x+y_{1}, y_{1}+y_{2}, \ldots, y_{k-1}+\right.$ $m\rangle \subseteq\langle T(M)\rangle$, we have $M=\left\langle x, x+y_{1}, y_{1}+y_{2}, \ldots, y_{k+1}+m\right\rangle$. Since $M$ is generated by $k+1$ elements of $T(M)$ and $\operatorname{diam}(T(\Gamma(M)))=n$, so $n \leq k+1$ by [14, Theorem 3.3]. Therefore $k \leq n \leq k+1$; so either $k=n$ or $k=n-1$ as required.

(2) Suppose that $n$ is an even integer. If $m-x=x$, then $d_{T_{0}}(m-x, x)=0$. So we assume that $m-x \neq x$. If $m \in T(M)$, then $M=\langle m\rangle \subseteq\langle T(M)\rangle$, this implies that $n=1$ [14, Theorem 3.3], a contradiction. So $d_{T_{0}}(m-x, x) \geq 2$. Let $x-y_{1}-y_{2}-\cdots-y_{k-1}-(m-x)$ be a shortest path from $x$ to $m-x$ in $T_{0}(\Gamma(M))$ of length $k$. Thus $k \leq n$. So $x+y_{1}, \ldots, y_{k-1}+(m-x) \in T(M)$. If $k$ is an odd integer, then $m=\left(x+y_{1}\right)-\left(y_{1}+y_{2}\right)+\cdots-\left(y_{k-2}+y_{k-1}\right)+\left(y_{k-1}+(m-x)\right)$. 
So we have $m \in\left\langle x+y_{1}, y_{1}+y_{2}, \ldots, y_{k-1}+(m-x)\right\rangle$. Then $M$ is generated by $k$ elements of $T(M)$. Thus $n \leq k$ by [14, Theorem 3.3]. So $k=n$ which is a contradiction. Hence $d_{T_{0}}(m-x, x)=k=d_{T}(m-x, x)$ for some even integer $k \leq n$.

(3) Let $n$ be an odd integer and $m+x \neq 0$. If $m+2 x \in T(M)$, then $d_{T_{0}}(m+x, x)=1$. So we may assume that $m+2 x \notin T(M)$ and then $d_{T_{0}}(m+$ $x, x) \geq 2$. Suppose that $x-y_{1}-y_{2}-\cdots-y_{k-1}-(m+x)$ be a shortest path from $x$ to $m+x$ in $T_{0}(\Gamma(M))$ of length $k$. Thus $k \leq n$. If $k$ is an even integer, then $-m=\left(x+y_{1}\right)-\left(y_{1}+y_{2}\right)+\cdots+\left(y_{k-2}+y_{k-1}\right)-\left(y_{k-1}+(m+x)\right)$. So $m \in\left\langle x+y_{1}, y_{1}+y_{2}, \ldots, y_{k-1}+(m+x)\right\rangle$. Then $M$ is generated by $k$ elements of $T(M)$. Thus $n \leq k$ by [14, Theorem 3.3]. Thus $k=n$ which is a contradiction. Hence $d_{T_{0}}(m+x, x)=k=d_{T}(m+x, x)$ for some odd integer $k \leq n$.

(4) Let $n$ be an integer and $x \in T(M)^{*}$. Then $0 \neq m-x \in M$ and $(m-x)+$ $x=m \notin T(M)$. So $m-x$ and $x$ are distinct. Therefore $k=d_{T_{0}}(m-x, x)$ is an even positive integer by part (2) above. Let $x-y_{1}-y_{2}-\cdots-y_{k-1}-(m-x)$ be a shortest path from $x$ to $m-x$ in $T_{0}(\Gamma(M))$ of length $k$. If $k<n$, then we have $m=2 x-\left(x+y_{1}\right)+\left(y_{1}+y_{2}\right)-\cdots-\left(y_{k-2}+y_{k-1}\right)+\left(y_{k-1}+(m-x)\right)$. So $m \in\left\langle x, x+y_{1}, y_{1}+y_{2}, \ldots, y_{k-1}+(m-x)\right\rangle$. Then $M$ is generated by $k+1$ elements of $T(M)$. Hence $n \leq k+1$. Thus $n=k+1$, which is a contradiction, since $n$ is an even integer but $k+1$ is an odd integer. So $d_{T_{0}}(m-x, x)=n=$ $d_{T}(m-x, x)$.

(5) Assume that $n$ is an odd integer and $x \in T(M)^{*}$. If $2 x+m \in T(M)$, then $m \in\langle x, 2 x+m\rangle$. So $M$ is generated by 2 elements of $T(M)$, this implies that $n=2$, which is a contradiction. Thus $0 \neq x+m, m \in M$ are distinct and $2 x+m \notin T(M)$. So $k=d_{T_{0}}(m+x, x) \geq 3$ is an odd integer by part (3) above. Let $x-y_{1}-y_{2}-\cdots-y_{k-1}-(m+x)$ be a shortest path from $x$ to $m+x$ in $T_{0}(\Gamma(M))$ of length $k$. if $k<n$, then $-m=2 x-\left(x+y_{1}\right)+\left(y_{1}+y_{2}\right)+\cdots+$ $\left(y_{k-2}+y_{k-1}\right)-\left(y_{k-1}+(m+x)\right)$. So $m \in\left\langle x, x+y_{1}, y_{1}+y_{2}, \ldots, y_{k-1}+(m+x)\right\rangle$ and $M$ is generated by $k+1$ elements of $T(M)$. Hence $n \leq k+1$. Thus $n=k+1$ which is a contradiction, since $n$ is an odd integer but $k+1$ is an even integer. So $d_{T_{0}}(m+x, x)=k=d_{T}(m+x, x)$.

Theorem 5.10. Let $M$ be a cyclic module over a commutative ring $R,|M| \geq 4$ and $T(M) \neq 0$. Then $\operatorname{diam}\left(T_{0}(\Gamma(M))\right)=\operatorname{diam}(T(\Gamma(M)))$.

Proof. Assume that $M$ is a cyclic module and $|M| \geq 4$. Then $T_{0}(\Gamma(M))$ is connected if and only if $T(\Gamma(M))$ is connected by Theorem 5.6 and $\operatorname{diam}\left(T_{0}(\Gamma(M))\right)$ $\leq \operatorname{diam}(T(\Gamma(M)))$ by Lemma 5.1. Thus $\operatorname{diam}(T(\Gamma(M)))=\infty$ if and only if $\operatorname{diam}\left(T_{0}(\Gamma(M))\right)=\infty$ by Theorem 5.8. So we may assume that $\operatorname{diam}(T(\Gamma(M)))$ $=n<\infty$. Let $M$ is generated by $m$ and $x \in T(M)^{*}$. If $n$ is an even integer, then $d_{T_{0}}(m-x, x)=d_{T}(m-x, x)=n$ by Lemma 5.9(4). If $n$ is an odd integer, then $d_{T_{0}}(m+x, x)=d_{T}(m+x, x)=n$ by Lemma 5.9(5), and hence $\operatorname{diam}\left(T_{0}(\Gamma(M))\right)=\operatorname{diam}(T(\Gamma(M)))=n$ by Lemma 5.1 . 
Recall that if $T(M)$ is not a submodule of $M$, then $\operatorname{gr}(\operatorname{Tor}(\Gamma(M)))=3$ or $\operatorname{gr}(\operatorname{Tor}(\Gamma(M)))=\infty$ and $\operatorname{gr}(T(\Gamma(M)))=3$ if and only if $\operatorname{gr}(\operatorname{Tor}(\Gamma(M)))=3$ [14, Theorem 3.5]. Also, If $\operatorname{gr}(T(\Gamma(M)))=4$, then $\operatorname{gr}(\operatorname{Tor}(\Gamma(M)))=\infty$ if $T(M)$ is not a submodule of $M[14$, Theorem 3.5]. Now, we provide a proof for the converse of [14, Theorem 3.5(3)] by torsion prime submodules of $M$.

Theorem 5.11. Let $M$ be a module over a commutative ring $R$ such that $T(M)$ is not a submodule of $M$. Then $\operatorname{gr}(T(\Gamma(M)))=4$, if and only if $\operatorname{gr}(\operatorname{Tor}(\Gamma(M)))=\infty$. Moreover, if $\operatorname{gr}(\operatorname{Tor}(\Gamma(M)))=\infty$, then $|T(M)|=3$.

Proof. If $\operatorname{gr}(T(\Gamma(M)))=4$, then $\operatorname{gr}(\operatorname{Tor}(\Gamma(M)))=\infty$ by [14, Theorem 3.5]. Conversely, suppose that $\operatorname{gr}(\operatorname{Tor}(\Gamma(M)))=\infty$. Since $T(M)$ is not a submodule of $M$, so $T(M) \neq M$. Then $T(M)=\bigcup_{\alpha \in \Lambda} L_{\alpha}$, where each $L_{\alpha}$ is a torsion prime submodule of $M$, then $|\Lambda| \geq 2$. If $\operatorname{gr}(\operatorname{Tor}(\Gamma(M)))=\infty$, then $x+y \in \operatorname{Tof}(M)$ for all distinct elements $x, y \in T(M)^{*}$. So $\left|L_{\alpha}\right|=2$ for every $\alpha \in \Lambda$. Hence the intersection of any two distinct $L_{\alpha}$ 's is $\{0\}$ and so $|\Lambda|=2$ by Proposition 2.4. So $T(M)=L_{1} \cup L_{2}$ for torsion prime submodules $L_{1}$ and $L_{2}$ of $M$ with $L_{1} \cap L_{2}=0$ and $\left|L_{1}\right|=\left|L_{2}\right|=2$. So we may assume that $L_{1}=\{0, x\}$ and $L_{2}=\{0, y\}$ where $2 x=2 y=0$. So $|T(M)|=3$ and $x+y \notin T(M)$. Thus $0-x-(x+y)-y-0$ is a 4 -cycle in $T(\Gamma(M))$. Then $\operatorname{gr}(T(\Gamma(M))) \leq 4$. Since $\operatorname{gr}(\operatorname{Tor}(\Gamma(M)))=\infty$, then $\operatorname{gr}(T(\Gamma(M)))=4$ by [14, Theorem 3.5].

The "moreover" statement follows directly from the above arguments.

Note that if $T(M)$ is a submodule of $M$, then $\operatorname{gr}(T(\Gamma(M)))=3$ if and only if $|T(M)| \geq 3, \operatorname{gr}(T(\Gamma(M)))=4$ if and only if $2 \notin Z(R)$ and $|T(M)|=2$, and $\operatorname{gr}(T(\Gamma(M)))=\infty$ otherwise [14, Theorem 2.9].

Also, if $T(M)$ is not a submodule of $M$, then $\operatorname{gr}(T(\Gamma(M)))=3$ if and only if $\operatorname{gr}(\operatorname{Tor}(\Gamma(M)))=3$ and $\operatorname{gr}(T(\Gamma(M)))=4$, if and only if $\operatorname{gr}(\operatorname{Tor}(\Gamma(M)))=\infty$ by [14, Theorem 3.5] and Theorem 5.11. Thus $\operatorname{gr}(T(\Gamma(M))) \in\{3,4, \infty\}$. The next theorem gives a more explicit description of $\operatorname{gr}(T(\Gamma(M)))$.

Theorem 5.12. Let $M$ be a module over a commutative ring $R$ and $T(M) \neq$ M. Then $\operatorname{gr}(T(\Gamma(M))) \in\{3,4, \infty\}$. Moreover,

(1) $\operatorname{gr}(T(\Gamma(M)))=3$ if and only if $\operatorname{gr}(\operatorname{Tor}(\Gamma(M)))=3$,

(2) $\operatorname{gr}(T(\Gamma(M)))=\infty$ if and only if either $M$ is a torsion free $R$-module or $2 \in Z(R)$ and $|T(M)|=2$,

(3) $\operatorname{gr}(T(\Gamma(M)))=4$ otherwise.

Proof. (1) This follows from [14, Theorems 2.9 and 3.5].

(2) Let $\operatorname{gr}(T(\Gamma(M)))=\infty$. Then $T(M)$ is a submodule of $M$ and $|T(M)| \leq 2$ by [14, Theorems 2.9 and 3.5] and Theorem 5.11. So either $|T(M)|=1$ or $|T(M)|=2$ and $2 \in Z(R)$ by [14, Theorem 2.9]. Then $M$ is a torsion free $R$-module or $2 \in Z(R)$ and $|T(M)|=2$.

Conversely, If $T(M)=0$, then $\operatorname{gr}(T(\Gamma(M)))=\infty$ by [14, Theorem 2.9]. So we assume that $2 \in Z(R)$ and $|T(M)|=2$. Let $T(M)=\{0, x\}$. So $2 x=0$, since $x \neq 0$ and $2 x \in T(M)$ by Lemma $2.3(5)$. Therefore $T(M)$ is a submodule of $M$. So $\operatorname{gr}(T(\Gamma(M)))=\infty$ by [14, Theorem 2.9]. 
Lemma 5.13. Let $M$ be a module over a commutative ring $R$.

(1) If $\operatorname{gr}\left(\right.$ Tor $\left._{0}(\Gamma(M))\right)=3$, then $\operatorname{gr}\left(T_{0}(\Gamma(M))\right)=\operatorname{gr}(T(\Gamma(M)))=3$.

(2) If $\operatorname{gr}(T(\Gamma(M)))=\infty$, then $\operatorname{gr}\left(T_{0}(\Gamma(M))\right)=\infty=\operatorname{gr}\left(\operatorname{Tor}_{0}(\Gamma(M))\right)$.

Proof. We have

$$
\operatorname{gr}(T(\Gamma(M))) \leq \operatorname{gr}\left(T_{0}(\Gamma(M))\right) \leq \operatorname{gr}\left(\operatorname{Tor}_{0}(\Gamma(M))\right),
$$

since $\operatorname{Tor}_{0}(\Gamma(M))$ is a (induced) subgraph of $T_{0}(\Gamma(M))$ and $T_{0}(\Gamma(M))$ is a (induced) subgraph of $T(\Gamma(M))$. So the proofs of (1) and (2) are clear.

Recall that if $R=Z_{6}$ and $M=R$ as an $R$-module, then $M$ is a $T$-reduced module and $\operatorname{gr}\left(\operatorname{Tor}_{0}(\Gamma(M))\right)=\infty$ by Example 4.3. But $\overline{1}-\overline{3}-\overline{5}-\overline{1}$ is a 3 -cycle in $T(\Gamma(M))$ and $T_{0}(\Gamma(M))$. So $\operatorname{gr}(T(\Gamma(M)))=\operatorname{gr}\left(T_{0}(\Gamma(M))\right)=3$. Therefore the converse of Lemma 5.13 is not true.

Now, we explicitly determine $\operatorname{gr}\left(T_{0}(\Gamma(M))\right)$. The proof breaks naturally into two cases depending on whether or not $M$ is a $T$-reduced $R$-module.

Theorem 5.14. Let $M$ be a non-T-reduced $R$-module. Then $g r\left(T_{0}(\Gamma(M))\right) \in$ $\{3,4, \infty\}$.

Proof. Let $M$ be a non-T-reduced $R$-module. Then $\operatorname{gr}\left(\operatorname{Tor}_{0}(\Gamma(M))\right) \in\{3, \infty\}$ by Theorem 4.1. If $\operatorname{gr}\left(\operatorname{Tor}_{0}(\Gamma(M))\right)=3$, then $\operatorname{gr}\left(T_{0}(\Gamma(M))\right)=3$ by Lemma 5.13. So we may assume that $\operatorname{gr}\left(\operatorname{Tor}_{0}(\Gamma(M))\right)=\infty$. Then $T(M)$ is the unique non-zero torsion prime submodule of $M$ with $|T(M)| \leq 3$ by Theorem 4.1. Now we split the proof into three cases:

Case 1. Assume that $|T(M)|=2$ and $2 \in Z(R)$, so $\operatorname{gr}(T(\Gamma(M)))=\infty$ by Theorem 5.12. Hence $\operatorname{gr}\left(T_{0}(\Gamma(M))\right)=\infty$ by Lemma 5.13 .

Case 2. If $|T(M)|=3$ and $2 \in Z(R)$, then $\operatorname{gr}(\operatorname{Tof}(\Gamma(M)))=3$ by [14, Theorem 2.9] and so $\operatorname{gr}\left(T_{0}(\Gamma(M))\right)=3$.

Case 3. If $|T(M)| \in\{2,3\}$ and $2 \notin Z(R)$, then

$$
\operatorname{gr}\left(T_{0}(\Gamma(M))\right) \leq \operatorname{gr}(\operatorname{Tof}(\Gamma(M)))=4
$$

by [14, Theorem 2.9] and since $\operatorname{Tof}(\Gamma(M))$ is a (induced) subgraph of $T_{0}(\Gamma(M))$. So $\operatorname{gr}\left(T_{0}(\Gamma(M))\right) \in\{3,4\}$.

Theorem 5.15. Let $M$ be a T-reduced $R$-module and $T(M) \neq 0, M$. Then $\operatorname{gr}\left(T_{0}(\Gamma(M))\right) \in\{3,4, \infty\}$.

Proof. If $\operatorname{gr}\left(\operatorname{Tor}_{0}(\Gamma(M))\right)=3$, then $\operatorname{gr}\left(T_{0}(\Gamma(M))\right)=3$ by Lemma 5.13. So we may assume that $\operatorname{gr}\left(\operatorname{Tor}_{0}(\Gamma(M))\right)=\infty$. Then $T(M)=L \cup K$ such that $L \cap K=\{0\}$ and $\max \{|L|,|K|\} \leq 3$ for some nonzero torsion prime submodule $L$ and $K$ of $M$ by Theorem 4.2. Then $T(M)$ is not a submodule of $M$ and $|T(M)| \in\{3,4,5\}$. Now we split the proof into three cases:

Case 1. If $|T(M)|=3$, then $L=\{0, x\}$ and $K=\{0, y\}$ of $M$ such that $2 x=$ $2 y=0$ and $x+y \notin T(M)$, since $L \cap K=0$. It is clear that $\operatorname{gr}(\operatorname{Tor}(\Gamma(M)))=$ $\infty$. So $\operatorname{gr}(T(\Gamma(M)))=4$ by Theorem 5.11. Hence $\operatorname{gr}(T(\Gamma(M)))=4 \leq$ $\operatorname{gr}\left(T_{0}(\Gamma(M))\right)$. If $2.1_{R} \neq 0$, then $2(x+y)=0$ implies that $x+y \in T(M)$ 
which is a contradiction. So $2.1_{R}=0$ and $2 m=0$ for every $m \in M$. If $|M|=4$, then it is clear that $\operatorname{gr}\left(T_{0}(\Gamma(M))\right)=\infty$. So we assume that $|M| \geq 5$. Then there is an element $z \in M \backslash T(M)$ such that $z \neq x+y$. Therefore $z-(z+x)-(z+x+y)-(z+y)-z$ is a 4-cycle in $T_{0}(\Gamma(M)$. Hence $\operatorname{gr}\left(T_{0}(\Gamma(M))\right)=4$.

Case 2. Suppose that $|T(M)|=4$. So $L=\left\{0, x_{1}, y_{1}\right\}$ and $K=\left\{0, x_{2}\right\}$. Then $x_{1}+y_{1} \in T(M), 2 x_{2}=0$ and $x_{1}+x_{2}, y_{1}+x_{2} \notin T(M)$ since $L \cap K=0$. So $\left(x_{2}+y_{1}\right)-x_{2}-\left(x_{1}+x_{2}\right)-\left(x_{2}+y_{1}\right)$ is a 3 -cycle in $T_{0}(\Gamma(M)$. Hence $\operatorname{gr}\left(T_{0}(\Gamma(M))\right)=3$.

Case 3. If $|T(M)|=5$, then $L=\left\{0, x_{1}, y_{1}\right\}$ and $K=\left\{0, x_{2}, y_{2}\right\}$. So $x_{1}+y_{1}=0$ and $2 x_{2}=y_{2}$. Then similarly $x_{1}-y_{1}-\left(x_{1}+x_{2}\right)-\left(x_{2}+y_{1}\right)-x_{1}$ is a 3 -cycle in $T_{0}\left(\Gamma(M)\right.$ and so $\operatorname{gr}\left(T_{0}(\Gamma(M))\right)=3$.

Therefore $\operatorname{gr}\left(T_{0}(\Gamma(M))\right) \in\{3,4, \infty\}$.

By Theorem 5.14 and Theorem 5.15, we have the following corollary:

Corollary 5.16. Let $M$ be a module over a commutative ring $R$. Then $\operatorname{gr}\left(T_{0}(\Gamma(M))\right) \in\{3,4, \infty\}$.

We end this paper with a theorem that shows the relationship of diameters and girths between $T_{0}(\Gamma(R))$ and $T_{0}(\Gamma(M))$ for an $R$-module $M$.

Theorem 5.17. Let $R$ be a commutative ring that is not an integral domain and let $M$ be an $R$-module.

(1) If $T_{0}(\Gamma(R))$ is connected, then $T_{0}(\Gamma(M))$ is connected. Moreover if $\operatorname{diam}\left(T_{0}(\Gamma(R))\right)=n$, then $\operatorname{diam}\left(T_{0}(\Gamma(M))\right) \leq 2 n+1$.

(2) If $M \neq T(M)$ and $\operatorname{gr}\left(T_{0}(\Gamma(R))\right) \in\{3,4\}$, then $\operatorname{gr}\left(T_{0}(\Gamma(M))\right) \in\{3,4\}$.

Proof. (1) Let $T_{0}(\Gamma(R))$ be connected and $m, n \in M^{*}$. Then there exists a path $z-a_{1}-a_{2}-\cdots-a_{k-1}-1$ from $z$ to 1 of length $k$ from $z$ to 1 for some $z \in Z(R)^{*}$. So $z, z+a_{1}, \ldots, a_{k-1}+1 \in Z(R)$. Thus $m-a_{k-1} m-\cdots-a_{1} m-$ $z m-z n-a_{1} n-\cdots-a_{k-1} n-n$ is a path from $m$ to $n$ of length at most $2 k+1$ by Lemma 2.3(5). The "moreover" statement follows directly from the above arguments and [6, Corollary 4.3].

(2) Let $\operatorname{gr}\left(T_{0}(\Gamma(R))\right)=3$. Then there is a 3 -cycle $r_{1}-r_{2}-r_{3}-r_{1}$ for nonzero distinct elements $r_{1}, r_{2}, r_{3} \in R$. Assume that $x \in M \backslash T(M)$. If $r_{i} x=r_{j} x$ for some $i, j=1,2,3$, then $\left(r_{i}-r_{j}\right) x=0$. Thus $r_{i}=r_{j}$, since $x \notin T(M)$. Therefore $r_{1} x, r_{2} x$ and $r_{3} x$ are distinct. So $r_{1} x-r_{2} x-r_{3} x-r_{1} x$ is a 3 -cycle in $T_{0}(\Gamma(M))$. If $\operatorname{gr}\left(T_{0}(\Gamma(R))\right)=4$, similarly one can show that $\operatorname{gr}\left(T_{0}(\Gamma(M))\right)=4$.

Example 5.18. Let $R=Z$ and $M=Z_{4}$ as an $R$-module. Since $R$ is an integral domain, so $\operatorname{gr}\left(T_{0}(\Gamma(R))\right)=\infty=\operatorname{diam}\left(T_{0}(\Gamma(R))\right)$ by $[6$, Theorem 4.8 and Example 4.6]. It is clear that $M$ is a torsion $R$-module and so $\operatorname{gr}\left(T_{0}(\Gamma(M))\right)=3$ and $\operatorname{diam}\left(T_{0}(\Gamma(M))\right)=1$. So the converses of (1) and (2) in Theorem 5.17, are not true.

Acknowledgments. The author is deeply grateful to the referee for careful reading of the manuscript and helpful suggestions. 


\section{References}

[1] A. Abbasi and Sh. Habibi, The total graph of a commutative ring with respect to proper ideals, J. Korean Math. Soc. 49 (2012), no. 1, 85-98.

[2] - The total graph of a module over a commutative ring with respect to proper submodules, J. Algebra Appl. 11 (2012), no. 3, 1250048, 13 pp.

[3] D. D. Anderson and S. Chun, The set of torsion elements of a module, Comm. Algebra. 42 (2014), 1835-1843.

[4] D. D. Anderson and M. Naseer, Beck's coloring of a commutative ring, J. Algebra. 159 (1993), no. 2, 500-514.

[5] D. F. Anderson, M. C. Axtell, and J. A. Stickles, Jr., Zero-divisor graphs in commutative rings, in Commutative Algebra, Noetherian and Non-Noetherian Perspectives (M. Fontana, S.-E. Kabbaj, B. Olberding, I. Swanson, Eds.), 23-45, Springer-Verlag, New York, 2011.

[6] D. F. Anderson and A. Badawi, On the total graph of a commutative ring without the zero element, J. Algebra Appl. 11 (2012), no. 4, 1250074, 18 pp.

[7] - The generalized total graph of a commutative ring, J. Algebra Appl. 12 (2013), no. 5, 1250212, 18 pp.

[8] ․ The total graph of a commutative ring, J. Algebra. 320 (2008), no. 7, 27062719.

[9] D. F. Anderson and P. F. Livingston, The zero-divisor graph of a commutative ring, J. Algebra. 217 (1999), no. 2, 437-447

[10] D. F. Anderson and S. B. Mulay, On the diameter and girth of a zero-divisor graph, J. Pure Appl. Algebra. 210 (2007), no. 2, 543-550.

[11] Z. Barati, K. Khashyarmanesh, F. Mohammadi, and K. Nafar, On the associated graphs to a commutative ring, J. Algebra Appl. 11 (2012), no. 2, 1250037, 17 pp.

[12] I. Beck, Coloring of commutative rings, J. Algebra. 116 (1988), no. 1, 208-226.

[13] S. Ebrahimi Atani and F. Esmaeili Khalil Saraei, The total torsion element graph of semimodules over commutative semirings, Algebra and Discrete Mathematics 16 (2013), no. 1, 1-15.

[14] S. Ebrahimi Atani and S. Habibi, The total torsion element graph of a module over a commutative ring, An. St. Univ. Ovidius Constant. 19 (2011), no. 1, 23-34.

[15] R. L. MacCasland and P. F. Smith , Prime submodules of Noetherian modules, Rocky Mountain J. Math. 23 (1993), 1041-1062.

FACULTY OF FOUMAN

College of Engineering

UNIVERSITY OF TEHRAN

P.O. Box 43515-1155, Fouman, Iran

E-mail address: f.esmaeili.kh@ut.ac.ir 